Fixed Point Theory, 20(2019), No. 1, 195-202

DOI: $10.24193 /$ fpt-ro.2019.1.12

http://www.math.ubbcluj.ro/ nodeacj/sfptcj.html

\title{
FIXED POINTS, MULTI-VALUED UNIFORMLY LIPSCHITZIAN MAPPINGS AND UNIFORM NORMAL STRUCTURE
}

\author{
JAROSŁAW GÓRNICKI \\ Department of Mathematics and Applied Physics \\ Rzeszów University of Technology \\ PO Box 85, 35-959 Rzeszów, Poland \\ E-mail: gornicki@prz.edu.pl
}

\begin{abstract}
Khamsi and Kirk [11] gave the definition of multi-valued uniformly Lipschitzian mappings via generalized orbits. We will prove a fixed point theorem in metric spaces with uniform normal structure which extends Lim and Xu's theorems [13] to multi-valued uniformly Lipschitzian mappings. We also give a fixed point result for multi-valued uniformly Lipschitzian mappings in hyperconvex spaces.
\end{abstract}

Key Words and Phrases: Fixed point, convexity structure, multi-valued mapping, normal structure coefficient, uniform normal structure.

2010 Mathematics Subject Classification: 47H10, 46B20.

\section{REFERENCES}

[1] R.P. Agarwal, D. O'Regan, D.R. Sahu, Fixed Point Theory for Lipschitzian-type Mappings with Applications, Springer Science and Business Media, Dordrecht 2009.

[2] J.M. Ayerbe Toledano, T. Domínguez Benavides, G. López Acedo, Measures of Noncompactness in Metric Fixed Point Theory, Birkhäuser Verlag, Basel 1997.

[3] J.B. Baillon, Quelques aspects de la théorie des points fixes dan les espaces de Banach I, in: Séminare d'Analyse Fonctionnelle de l'École Polytechnique VII (1978-1979), École Polytechnique, Palaiseau, 1979.

[4] W.L. Bynum, Normal structure coefficients for Banach spaces, Pacific J. Math., 86(1980), 427-436.

[5] E. Casini, E. Maluta, Fixed points of uniformly Lipschitzian mappings in spaces with uniformly normal structure, Nonlinear Anal., 9(1985), 103-108.

[6] T. Domínguez Benavides, Fixed point theorems for uniformly Lipschitzian mappings and asymptotically regular mappings, Nonlinear Anal., 32(1998), 15-27.

[7] N. Dunford, J.T. Schwartz, Linear Operators, Part 1, Interscience, New York 1958.

[8] R. Espínola, M.A. Khamsi, Introduction to hyperconvex spaces, in: W.A. Kirk, B. Sims (eds.), Handbook of Metric Fixed Point Theory, Kluwer, Dordrecht 2001, 391-435.

[9] K. Goebel, W.A. Kirk, A fixed point theorem for transformations whose iterates have uniform Lipschitz constant, Studia Math., 47(1973), 135-140.

[10] J. Górnicki, Fixed point theorems for multi-valued uniformly Lipschitzian mappings in Banach and metric spaces, J. Nonlinear Convex Anal., 17(2016), 2455-2467. 
[11] M.A. Khamsi, W.A. Kirk, On uniformly Lipschitzian multivalued mappings in Banach and metric spaces, Nonlinear Anal., 72(2010), 2080-2085.

[12] E.A. Lifshitz, A fixed point theorem for operators in strongly convex spaces, (Russian), Voronez. Gos. Univ. Trudy Mat. Fak., 16(1975), 23-28.

[13] T.C. Lim, H.K. Xu, Uniformly Lipschitzian mappings in metric spaces with uniform normal structure, Nonlinear Anal., 25(1995), 1231-1235.

[14] S.B. Nadler, Multi-valued contraction mappings, Pacific J. Math., 30(1969), 475-488.

Received: September 22, 2016; Accepted: April 21, $201 \%$. 
FIXED POINTS, MULTI-VALUED UNIFORMLY LIPSCHITZIAN MAPPINGS 